\title{
High-precision analysis of binary stars with planets
}

\section{Searching for condensation temperature trends in the HD 106515 system $\star$}

\author{
C. Saffe ${ }^{1,2,5}$, E. Jofré ${ }^{3,5,6}$, P. Miquelarena ${ }^{2,5}$, M. Jaque Arancibia ${ }^{4}$, M. Flores ${ }^{1,2,5}$, F. M. López ${ }^{1,2,5}$, and A. Collado ${ }^{1,2,5}$ \\ ${ }^{1}$ Instituto de Ciencias Astronómicas, de la Tierra y del Espacio (ICATE-CONICET), C.C 467, 5400 San Juan, Argentina \\ e-mail: csaffe@conicet.gov.ar \\ ${ }^{2}$ Facultad de Ciencias Exactas, Universidad Nacional de San Juan (UNSJ), Físicas y Naturales (FCEFN), San Juan, Argentina \\ ${ }^{3}$ Observatorio Astronómico de Córdoba (OAC), Laprida 854, X5000BGR Córdoba, Argentina \\ ${ }^{4}$ Departamento de Física y Astronomía, Universidad de La Serena, Av. Cisternas 1200. 1720236 La Serena, Chile \\ ${ }^{5}$ Consejo Nacional de Investigaciones Científicas y Técnicas (CONICET), Argentina \\ ${ }^{6}$ Instituto de Astronomía, Universidad Nacional Autónoma de México, Ciudad Universitaria, Ciudad de México 04510, México
}

Received 24 February 2019 / Accepted 1 April 2019

\begin{abstract}
Aims. We explore for the first time the probable chemical signature of planet formation in the remarkable binary system HD 106515 . Star A hosts a massive long-period planet with $\sim 9 M_{\text {Jup }}$ detected by radial velocity, while there is no planet detected at the B star. We also refine stellar and planetary parameters by using non-solar-scaled opacities when modelling the stars.

Methods. We carried out a simultaneous determination of stellar parameters and abundances by applying for the first time non-solarscaled opacities in this binary system, in order to reach the highest possible precision. We used a line-by-line strictly differential approach, using the Sun and then the A star as reference. Stellar parameters were determined by imposing an ionization and excitation balance of Fe lines, with an updated version of the FUNDPAR program, ATLAS12 model atmospheres, and the MOOG code. Opacities for an arbitrary composition were calculated through the opacity sampling method. The chemical patterns were compared with solartwins condensation temperature $T_{\mathrm{c}}$ trends from the literature and also mutually between both stars. We take the opportunity to compare and discuss the results of the classical solar-scaled method and the high-precision procedure applied here.

Results. Stars A and B in the binary system HD 106515 do not seem to be depleted in refractory elements, which is different when comparing the Sun with solar twins. The terrestrial planet formation would have been less efficient in the stars of this binary system. Together with HD 80606/7, this is the second binary system that does not seem to present a (terrestrial) signature of planet formation, when both systems host an eccentric giant planet. This is in agreement with numerical simulations, where the early dynamical evolution of eccentric giant planets clears out most of the possible terrestrial planets in the inner zone. We refined the stellar mass, radius, and age for both stars and found a notable difference of $\sim 78 \%$ in $R_{\star}$ compared to previous works. We also refined the planet mass to $m_{\mathrm{p}} \sin i=$ $9.08 \pm 0.20 M_{\text {Jup }}$, which differs by $\sim 6 \%$ compared with the literature. In addition, we showed that the non-solar-scaled solution is not compatible with the classical solar-scaled method, and some abundance differences are comparable to non-local thermodynamic equilibrium (NLTE) or galactic chemical evolution (GCE) effects especially when using the Sun as reference. Therefore, we encourage the use of non-solar-scaled opacities in high-precision studies such as the detection of $T_{\mathrm{c}}$ trends.
\end{abstract}

Key words. stars: abundances - planetary systems - binaries: visual - stars: individual: HD 106515

\section{Introduction}

In recent years, the achieved high precision in the derivation of stellar parameters and chemical abundances has allowed us to study in detail possible differences in stars with and without planets (e.g. Meléndez et al. 2009; Ramírez et al. 2011; Bedell et al. 2014; Saffe et al. 2017). For instance, the search for planet formation or accretion signatures in the photospheric composition of the stars was performed by looking at the condensation temperature $T_{\mathrm{c}}$ trends, with an unprecedented dispersion in metallicity below $\sim 0.01$ dex (e.g. Meléndez et al. 2009; Liu et al. 2014; Saffe et al. 2015, 2016, 2017). In particular, Meléndez et al. (2009; hereafter M09) detected a deficiency in refractory elements in the Sun with respect to 11 solar twins, suggesting that

\footnotetext{
^ The data presented herein were obtained at the W.M. Keck Observatory, which is operated as a scientific partnership between the California Institute of Technology, the University of California, and the National Aeronautics and Space Administration. The Observatory was made possible by the generous financial support of the W.M. Keck Foundation.
}

the refractory elements depleted in the solar photosphere are possibly trapped in terrestrial planets and/or in the cores of giant planets. The same conclusion was also reached by Ramírez et al. (2009, 2010), using larger samples of solar twins and analogues.

The study of binary or multiple systems plays a central role in the detection of the possible chemical signature of planet formation, providing that the stars were born from the same molecular cloud. Differential abundances between the components of these systems greatly diminishes effects such as galactic chemical evolution (GCE) or the galactic birth place of the stars, which could affect $T_{\mathrm{c}}$ trends (see e.g. González Hernández et al. 2013; Adibekyan et al. 2014, 2016). In addition, the analysis of two physically similar stars using one of them as a reference star allows us to diminish the dispersion in both the derivation of stellar parameters and chemical abundances (e.g. Saffe et al. 2015). Therefore, a binary system with similar stellar components where only one of them is orbited by a planet is an ideal laboratory to look for very small chemical differences that could be attributed to the planet formation process. 
To date, although more than $\sim 2990$ planetary systems are known ${ }^{1}$, to find these kind of systems has proven to be a very difficult task. Examples of these binary systems previously studied in the literature include 16 Cyg, HAT-P-1, HD 80606, and HAT-P-4 (e.g. Ramírez et al. 2011; Liu et al. 2014; Saffe et al. $2015,2017)$. There are also binary systems in which circumstellar planets orbit both stars of the system, such as HD 20781, HD 133131, and WASP-94 (Mack et al. 2014; Teske et al. 2016a,b). Then, there is a need for additional stars hosting planets in binary systems to be compared through a high-precision abundance determination. Due to their importance, some of these unique systems such as 16 Cyg received the attention of many different works studying their chemical composition in detail (e.g. Laws \& Gonzalez 2016; Takeda 2005; Schuler et al. 2011; Ramírez et al. 2011; Tucci Maia et al. 2014). Some works suggested that both stars present the same chemical composition (Takeda 2005; Schuler et al. 2011) while other studies found that 16 Cyg A is more metal rich than the planet host B component (Laws \& Gonzalez 2016; Ramírez et al. 2011; Tucci Maia et al. 2014; Nissen et al. 2017). In addition, the complete $T_{\mathrm{c}}$ trend detected by Tucci Maia et al. (2014) between the stars of 16 Cyg covers a range of only 0.04 dex between the maximum and minimum abundance values of 19 different chemical species (see their Fig. 3). More recently, Nissen et al. (2017) performed a high-precision analysis of this pair using High Accuracy Radial velocity Planet Searcher for the Northern hemisphere (HARPS-N) spectra and found a clear trend with $T_{\mathrm{c}}$. These examples show that the detection of a chemical difference or a possible $T_{\mathrm{c}}$ trend as a chemical signature of planet formation is a challenge, and does require the maximum precision in both stellar parameters and abundances (for a more complete discussion, see also Saffe et al. 2018). Recently, our group achieved a major step forward in the pursuit of the highest possible precision. For the first time, we used non-solar-scaled opacities in a simultaneous derivation of both stellar parameters and abundances (Saffe et al. 2018) for main sequence and giant stars. When modelling the atmosphere of the stars, the four stellar parameters usually taken as $T_{\text {eff }}, \log g,[\mathrm{Fe} / \mathrm{H}]$, and $v_{\text {micro }}$ are now taken as $T_{\text {eff }}, \log g$, chemical pattern, and $v_{\text {micro }}$. In this way, we showed that many chemical species show a small but noticeable variation when using the new doubly iterated method instead of the usual solar-scaled methods, implying that $T_{\mathrm{c}}$ trends could also vary. We started a new programme in order to detect the possible chemical signature of planet formation in these binary systems by taking advantage of this improvement in the technique.

Mayor et al. (2011) first announced through a preprint the detection of a high-mass giant planet orbiting the star HD 106515 A with a period of $\sim 9.9 \mathrm{yr}$ and a minimum mass $\mathrm{m} \sin i \sim 10 M_{\mathrm{Jup}}$, as a part of a HARPS radial velocity (RV) survey. The true mass of this object could correspond to the transition region between planets and brown dwarfs. The host star belongs to a wide binary system together with HD $106515 \mathrm{~B}$, separated by $7.5 \operatorname{arcsec}(\sim 250 \mathrm{AU})$, in which both stars have a similar mass and a metallicity close to solar (Desidera et al. $2004,2006)$. The presence of this massive planet was later confirmed in the work of Desidera et al. (2012), who performed a RV monitoring of both stars in this system using Spettrografo Alta Risoluzione Galileo (SARG) spectra. However, the authors do not find significant RV variations on the B star, and rule out additional stellar companions by using adaptive optics images. They propose that the relatively high eccentricity of the

http://exoplanet.eu/catalog/ planet $(0.572 \pm 0.011)$ may arise from the Kozai mechanism, that is, a dynamical perturbation due to the presence of the wide stellar component. Marmier et al. (2013) updated some orbital parameters of the planet by using CORALIE ${ }^{2}$ spectra, and proposed a possible Kozai mechanism similar to Desidera et al. (2012). We note that this binary system is remarkable for a number of reasons. First, there is a notable similarity between the stars of this system, which show an estimated difference in effective temperature and superficial gravity of $+157 \pm 11 \mathrm{~K}$ and $-0.02 \pm 0.15$ dex (Desidera et al. 2004), taken as A - B. This makes HD 106515 a unique target to analyse through a differential study, belonging to the select group of binary systems with similar components and having a planet orbiting only one star. Second, from more than $\sim 2990$ planetary systems detected, only 28 of them $(<0.1 \%)$ are known with a period greater than $9 \mathrm{yr}$. The long period coverage is very important in order to properly constrain models of planet formation and migration. In addition, with a mass higher than $6 M_{\text {Jup }}$, this planet belong to the upper $\sim 15 \%$ of the planetary mass distribution (see e.g. Marmier et al. 2013). Therefore, the study of this object give us the possibility, for the first time, to test the possible chemical signature of planet formation for the case of a high-mass longperiod planet. We take advantage of our recent improvement in the derivation of high-precision abundances (Saffe et al. 2018) to study this notable binary system with a line-by-line differential approach, aiming to detect a slight contrast between the components.

This work is organized as follows. In Sect. 2 we describe the observations and data reduction, while in Sect. 3 we present the stellar parameters and chemical abundance analysis, and present a refined value for the planetary mass. In Sect. 4 we show the results and discussion, and finally in Sect. 5 we highlight our main conclusions.

\section{Observations and data reduction}

Observations of the HD 106515 binary system were acquired using the High Resolution Echelle Spectrometer (HIRES, Vogt et al. 1994) attached to the right Nasmyth platform of the Keck $10-\mathrm{m}$ telescope on Mauna Kea, Hawaii. HIRES is a grating crossdispersed echelle spectrograph, equipped with a $2048 \times 4096$ Massachusets Institute of Technology-Lincoln Laboratory (MIT-LL) detector with a pixel size of $15 \mu \mathrm{m}$. The stellar spectra for this work were downloaded from the Keck Observatory Archive $(\mathrm{KOA})^{3}$, under the program ID N158Hr. The slit used was B2 with a width of 0.574 arcsec, which provides a measured resolution of $\sim 67000$ at $\sim 5200 \AA^{4}$.

The observations were taken on December 9, 2013, with the B star observed immediately after the A star, using the same spectrograph configuration for both objects. The exposure times were 180 and $240 \mathrm{~s}$ on each target, obtaining a final signalto-noise ratio $(\mathrm{S} / \mathrm{N})$ of $\sim 300$ measured at $\sim 6000 \AA$. The final spectral coverage is $\sim 4700-8900 \AA$. The asteroid Iris was also observed with the same spectrograph set-up, achieving a similar $\mathrm{S} / \mathrm{N}$, to acquire the solar spectrum useful for reference in our (initial) differential analysis. We note, however, that the final differential study with the highest abundance precision is between stars A and B because of their high degree of similarity.

2 CORALIE is not an actual acronym. CORALIE was instead named after the baby daughter of an engineer from the Observatoire de HauteProvence in France.

3 http://www2.keck .hawaii.edu/koa/koa.html

4 http://www2.keck.hawaii.edu/inst/hires/slitres.html 
HIRES spectra were reduced using the data reduction package $\mathrm{MAKEE}^{5}$ (MAuna Kea Echelle Extraction), which performs the usual reduction process including bias subtraction, flat fielding, spectral order extractions, and wavelength calibration. The continuum normalization and other operations (such as Doppler correction) were performed using Image Reduction and Analysis Facility (IRAF) ${ }^{6}$.

\section{Stellar parameters and chemical abundance analysis}

We derived the fundamental parameters $\left(T_{\text {eff }}, \log g\right.$, chemical pattern, $v_{\text {micro }}$ ) of stars $\mathrm{A}$ and $\mathrm{B}$ following the same procedure detailed in our previous work (Saffe et al. 2018). We started by measuring the equivalent widths (EW) of Fe I and Fe II lines in the spectra of our programme stars using the IRAF task splot, and then continued with other chemical species. The lines list and relevant laboratory data were taken from Liu et al. (2014), Meléndez et al. (2014), and then extended with data from Bedell et al. (2014), who carefully selected lines for a high-precision abundance determination.

Stellar parameters and abundances were derived simultaneously by imposing excitation and ionization balance of $\mathrm{Fe} \mathrm{I}$ and $\mathrm{Fe}$ II lines. We used an updated version of the program FUNdamental PARameters (FUNDPAR, Saffe 2011; Saffe et al. 2018), which uses the MOOG code (Sneden 2016) together with ATLAS12 model atmospheres (Kurucz 1993) to search for the appropriate solution. The procedure uses explicitly calculated (i.e. non-interpolated) plane-parallel local thermodynamic equilibrium (LTE) Kurucz's model atmospheres, including the internal calculation of specific opacities through the opacity sampling (OS) method. The first FUNDPAR iterative process searches the iron balance with the usual solar-scaled model atmospheres. A starting set of parameters and abundances is determined using EWs and spectral synthesis. Then, the iterative process in FUNDPAR is restarted, but using ATLAS12 model atmospheres scaled to the last set of abundances found. This new iteration includes the calculation of specific opacities for the last chemical pattern specified, and not only a mere change in the abundances of the model. Thus, ATLAS 12 models are described as $\left(T_{\text {eff }}, \log g\right.$, chemical pattern, $\left.v_{\text {micro }}\right)$ rather than the usual solar-scaled $\left(T_{\text {eff }}, \log g,[\mathrm{Fe} / \mathrm{H}], v_{\text {micro }}\right)$. New stellar parameters and abundances are then successively derived, finishing the process consistently when the stellar parameters are the same as the previous step (for more details, see Saffe et al. 2018).

Stellar parameters of stars A and B were determined by applying the full ${ }^{7}$ line-by-line differential technique, using the Sun as standard in an initial approach, and then we recalculated the parameters of the B star using A as reference. Firstly, we derived absolute abundances for the Sun using $5777 \mathrm{~K}$ for $T_{\text {eff }}$, 4.44 dex for $\log g$ and an initial $v_{\text {turb }}$ of $1.0 \mathrm{~km} \mathrm{~s}^{-1}$. Then, the solar $v_{\text {turb }}$ was estimated by requiring zero slope in the absolute abundances of Fe I lines versus $\mathrm{EW}_{\mathrm{r}}$ and obtained a final $v_{\text {turb }}$ of $0.91 \mathrm{~km} \mathrm{~s}^{-1}$. We note, however, that the exact values are not crucial for our strictly differential study (see e.g. Bedell et al. 2014; Saffe et al. 2015).

\footnotetext{
5 http://www . astro.caltech.edu/ tb/makee/

6 IRAF is distributed by the National Optical Astronomical Observatories, which is operated by the Association of Universities for Research in Astronomy, Inc. under a cooperative agreement with the National Science Foundation.

7 By "full" we mean that line-by-line differences were considered in both the derivation of stellar parameters and (not only) abundances.
}
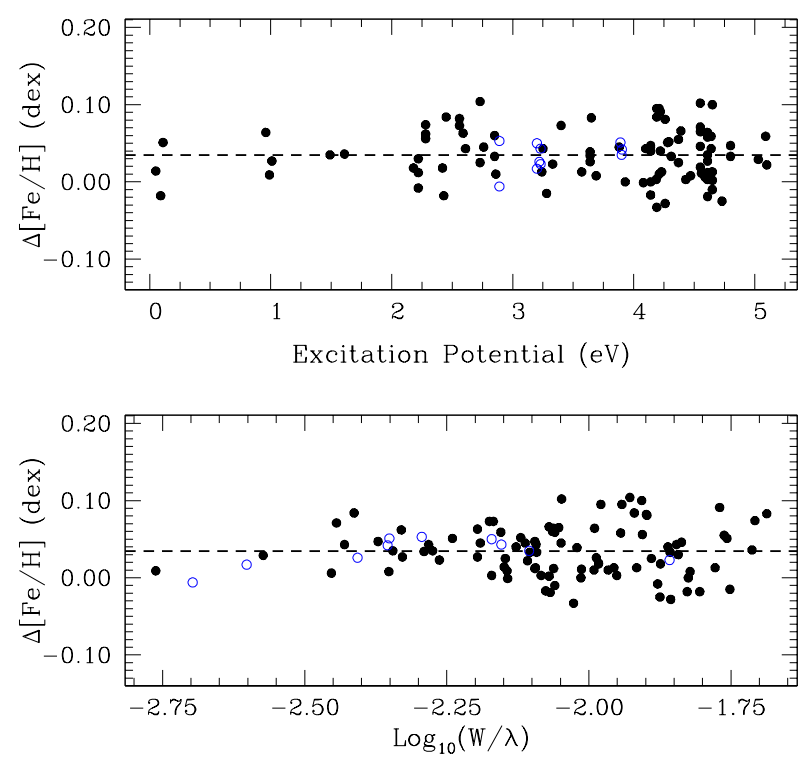

Fig. 1. Differential abundance versus excitation potential (upper panel) and differential abundance versus reduced EW (lower panel) for star A relative to the Sun. Filled and empty points correspond to Fe I and Fe II, respectively. The dashed line is a linear fit to the abundance values.

The next step was the determination of stellar parameters of stars A and B using the Sun as standard, that is, (A - Sun) and (B - Sun). The resulting stellar parameters for star A were $T_{\text {eff }}=5364 \pm 57 \mathrm{~K}, \log g=4.39 \pm 0.18 \mathrm{dex},[\mathrm{Fe} / \mathrm{H}]=+0.016 \pm$ 0.009 dex, and $v_{\text {turb }}=0.79 \pm 0.12 \mathrm{~km} \mathrm{~s}^{-1}$. For star B we obtained $T_{\text {eff }}=5190 \pm 58 \mathrm{~K}, \log g=4.30 \pm 0.20 \mathrm{dex},[\mathrm{Fe} / \mathrm{H}]=+0.022 \pm$ $0.010 \mathrm{dex}$, and $v_{\text {turb }}=0.58 \pm 0.15 \mathrm{~km} \mathrm{~s}^{-1}$. The errors in the stellar parameters were derived following the procedure detailed in Saffe et al. (2015), which takes into account the individual and the mutual covariance terms of the error propagation. We present in Figs. 1 and 2 abundance versus excitation potential and abundance versus reduced equivalent width $\left(\mathrm{EW}_{\mathrm{r}}\right)$ for both stars. Filled and empty points correspond to Fe I and Fe II, while the dashed lines are linear fits to the differential abundance values.

The stellar parameters and abundances of the B star were then redetermined, but using the A star as reference instead of the Sun $(B-A)$ to perform the differential analysis. Similar to previous works, we chose the hotter star of the pair as reference (e.g. Saffe et al. 2015, 2016, 2017). Figure 3 shows the plots of abundance versus excitation potential and abundance versus $\mathrm{EW}_{\mathrm{r}}$, using similar symbols to those used in Figs. 1 and 2. The resulting stellar parameters for star B are the same as those obtained when we used the Sun as a reference, $T_{\text {eff }}=5190 \pm 48 \mathrm{~K}, \log g=4.30 \pm 0.17 \mathrm{dex},[\mathrm{Fe} / \mathrm{H}]=+0.022 \pm$ $0.009 \mathrm{dex}$, and $v_{\text {turb }}=0.58 \pm 0.12 \mathrm{~km} \mathrm{~s}^{-1}$.

We computed the individual abundances for the following elements: C I, O I, Na I, Mg I, Al I, Si I, S I, Ca I, Sc I, Sc II, Ti I, Ti II, V I, Cr I, Cr II, Mn I, Fe I, Fe II, Co I, Ni I, Cu I, Sr I, Y II, and $\mathrm{Ba}$ II. The Li I line $6707.8 \AA$ is not present in the spectra. The hyperfine structure splitting (HFS) was considered for V I, Mn I, Co I, CuI, and Ba II by adopting the HFS constants of Kurucz \& Bell (1995) and performing spectral synthesis with the program MOOG (Sneden 2016) for these species. The same spectral lines were measured in both stars. We applied non-local thermodynamic equilibrium (NLTE) corrections to the O I triplet following Ramírez et al. (2007). The abundances for O I (NLTE) are lower than LTE values $(\sim 0.15$ and $\sim 0.14$ dex for stars A and B). The forbidden [O I] lines at 6300.31 and $6363.77 \AA$ are 

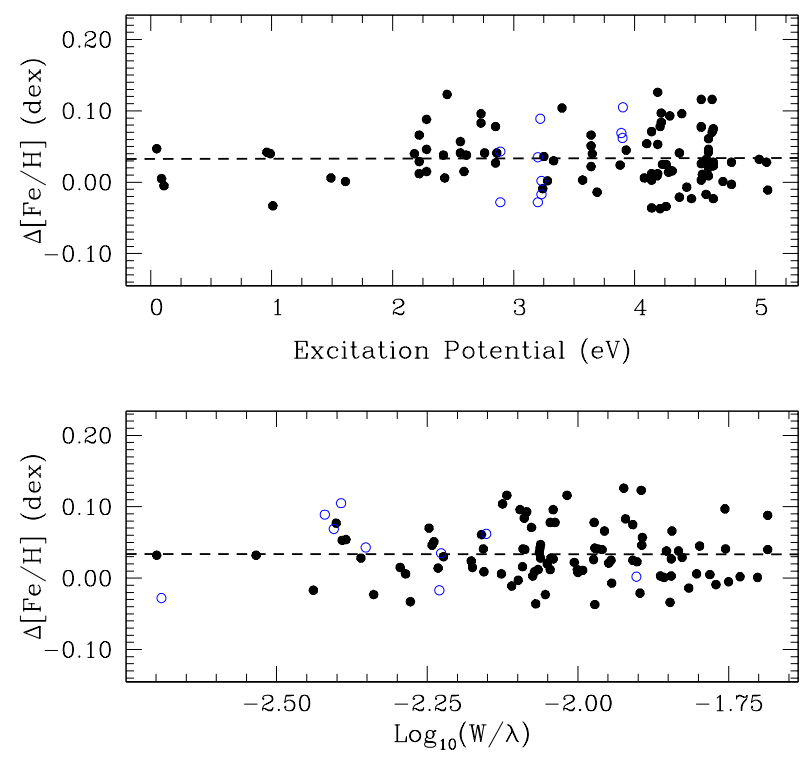

Fig. 2. Differential abundance versus excitation potential (upper panel) and differential abundance versus reduced EW (lower panel) for star B relative to the Sun. Filled and empty points correspond to Fe I and Fe II, respectively. The dashed line is a linear fit to the abundance values.
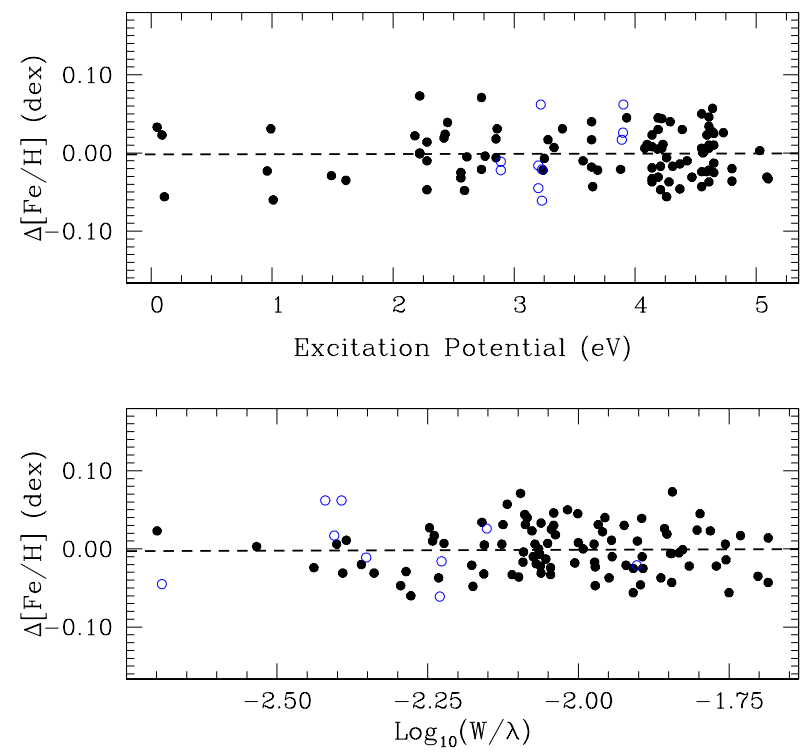

Fig. 3. Differential abundance versus excitation potential (upper panel) and differential abundance versus reduced EW (lower panel) for star B relative to A (B - A). Filled and empty points correspond to Fe I and Fe II, respectively. The dashed line is a linear fit to the data.

weak in our stars. Both [O I] lines are blended in the solar spectra: with two N I lines in the red wing of [O I] $6300.31 \AA$ and with CN near [O I] $6363.77 \AA$ (Lambert 1978; Johansson et al. 2016; Bensby et al. 2004). Therefore, we prefer to avoid these weak [O I] lines in our calculation and only use the O I triplet. We also applied NLTE corrections to Ba II following Korotin et al. (2015), obtaining NLTE values slightly lower than LTE ( $\sim 0.04$ dex and $\sim 0.03$ dex for stars A and B), and NLTE corrections to Na following Shi et al. (2004), estimating in this case NLTE values lower than LTE by $\sim 0.05$ dex for both stars. As expected, NLTE corrections were very similar for both objects, which is convenient for the differential analysis.
The final differential abundances $[\mathrm{X} / \mathrm{Fe}]^{8}$ for (A - Sun), (B - Sun), and (B - A) are presented in Table 1. Similar to previous works, we present for each species the observational error $\sigma_{\text {obs }}$ (estimated as $\sigma / \sqrt{(n-1)}$, where $\sigma$ is the standard deviation of the different lines) as well as systematic errors due to uncertainties in the stellar parameters $\sigma_{\text {par }}$ (by adding quadratically the abundance variation when modifying the stellar parameters by their uncertainties). For those chemical species with only one line, we adopted for $\sigma$ the average standard deviation of the other elements. The total error $\sigma_{\text {TOT }}$ was obtained by quadratically adding $\sigma_{\mathrm{obs}}, \sigma_{\mathrm{par}}$, and the error in $[\mathrm{Fe} / \mathrm{H}]$.

From our new atmospheric parameters in combination with $V$ magnitudes Gaia DR2 (Gaia Collaboration 2018) parallaxes and stellar evolutionary models, we derived refined stellar mass $M_{\star}$, radius $R_{\star}$, and age $\tau_{\star}$ for HD $106515 \mathrm{~A}$ and $\mathrm{B}$. We employed a Bayesian estimation method and PAdova and TRieste Stellar Evolution Code (PARSEC) isochrones (Bressan et al. 2012) via web interface PARAM $1.3^{9}$ (da Silva et al. 2006). We obtain $M_{\star}=0.888 \pm 0.018 M_{\odot}, R_{\star}=0.910 \pm 0.009 R_{\odot}, \tau_{\star}=$ $9.233 \pm 2.133 \mathrm{Gyr}$ and $M_{\star}=0.861 \pm 0.015 M_{\odot}, R_{\star}=0.865 \pm$ $0.015 R_{\odot}, \tau_{\star}=9.155 \pm 2.199 \mathrm{Gyr}$ for $\mathrm{HD} 106515 \mathrm{~A}$ and $\mathrm{B}$, respectively. These stellar masses and radii imply stellar densities of $\rho_{\star}=1.66 \pm 0.05 \mathrm{~g} \mathrm{~cm}^{-3}$ and $\rho_{\star}=1.88 \pm 0.06 \mathrm{~g} \mathrm{~cm}^{-3}$ for the A and B component, respectively. Our estimations of mass are in good agreement with the values derived by Desidera et al. (2006), who used the same Bayesian method although using isochrones from Girardi et al. (2000). Estimations of radii are not reported in Desidera et al., however, our radii are in perfect agreement with those provided by Gaia DR2.

On the other hand, for HD 106515 A Marmier et al. (2013) derived $M_{\star}=0.97 \pm 0.01 M_{\odot}$ and $R_{\star}=1.62 \pm 0.05 R_{\odot}$. The masses agree only within $3 \sigma$, however their radius is $78 \%$ larger than our estimation. Although they also employed the PARAM code, but with the stellar models of Girardi et al. (2000), we noticed that for this star they reported a magnitude of $V=7.35$ taken from the High Precision Parallax Collecting Satellite (HIPPARCOS) catalogue (ESA 1997), which is considerably different from the one we employed from the Tycho-2 catalogue $(V=7.97$, Høg et al. 2000) and that is displayed on Set of Identifications, Measurements and Bibliography for Astronomical Data (SIMBAD). This is probably the main reason for the discrepancies with our stellar parameters, especially radius.

Finally, combining our refined mass estimation of HD 106515 A with the parameters from the spectroscopic orbit (velocity semi-amplitude $K$, period $P$, eccentricity $e$ ) of Marmier et al. (2013), we derived an improved value of the minimum mass $m_{\mathrm{p}} \sin i$ of HD 106515 Ab. Using Eq. (1) from Cumming et al. (1999), we derive $m_{\mathrm{p}} \sin i=9.08 \pm 0.20 M_{\text {Jup }}$, which is $\sim 6 \%$ $\left(\sim 175 M_{\oplus}\right)$ smaller than the value reported by Marmier et al. (2013) of $m_{\mathrm{p}} \sin i=9.61 \pm 0.14 M_{\mathrm{Jup}}{ }^{10}$. We present in Table 2 the stellar and planetary parameters derived in this work.

\section{Results and discussion}

The differential abundances of stars A and B relative to the Sun are presented in Figs. 4 and 5. We took the condensation temperatures from the $50 \% T_{\mathrm{c}}$ values derived by Lodders (2003). The chemical comparison between one star and the Sun could be affected by GCE effects because of their different (chemical)

\footnotetext{
8 We used the standard notation $[\mathrm{X} / \mathrm{Fe}]=[\mathrm{X} / \mathrm{H}]-[\mathrm{Fe} / \mathrm{H}]$.

9 http://stev.oapd.inaf.it/cgi-bin/param_1.3

${ }^{10}$ Value currently reported in The Extrasolar Planets Encyclopaedia.
} 
Table 1. Differential abundances for stars A and B relative to the Sun, and B relative to A: (A - Sun), (B - Sun), and (B - A).

\begin{tabular}{|c|c|c|c|c|c|c|c|c|c|c|c|c|}
\hline \multirow[b]{2}{*}{ Element } & \multicolumn{4}{|c|}{$(\mathrm{A}-\mathrm{Sun})$} & \multicolumn{4}{|c|}{ (B - Sun) } & \multicolumn{4}{|c|}{$(B-A)$} \\
\hline & {$[\mathrm{X} / \mathrm{Fe}]$} & $\sigma_{\text {obs }}$ & $\sigma_{\mathrm{par}}$ & $\sigma_{\mathrm{TOT}}$ & {$[\mathrm{X} / \mathrm{Fe}]$} & $\sigma_{\mathrm{obs}}$ & $\sigma_{\mathrm{par}}$ & $\sigma_{\mathrm{TOT}}$ & {$[\mathrm{X} / \mathrm{Fe}]$} & $\sigma_{\mathrm{obs}}$ & $\sigma_{\mathrm{par}}$ & $\sigma_{\mathrm{TOT}}$ \\
\hline C I & 0.336 & 0.025 & 0.077 & 0.081 & 0.384 & 0.038 & 0.088 & 0.096 & 0.047 & 0.020 & 0.074 & 0.078 \\
\hline O I & -0.005 & 0.011 & 0.054 & 0.056 & 0.023 & 0.038 & 0.062 & 0.073 & 0.026 & 0.027 & 0.052 & 0.059 \\
\hline $\mathrm{Na} I$ & 0.038 & 0.045 & 0.030 & 0.055 & 0.024 & 0.052 & 0.035 & 0.064 & -0.014 & 0.013 & 0.029 & 0.033 \\
\hline Mg I & 0.105 & 0.016 & 0.025 & 0.031 & 0.086 & 0.024 & 0.025 & 0.036 & -0.020 & 0.019 & 0.021 & 0.030 \\
\hline $\mathrm{Al} \mathrm{I}$ & 0.197 & 0.040 & 0.039 & 0.056 & 0.176 & 0.049 & 0.044 & 0.067 & -0.021 & 0.008 & 0.038 & 0.040 \\
\hline Si I & 0.094 & 0.008 & 0.003 & 0.012 & 0.099 & 0.008 & 0.005 & 0.014 & 0.005 & 0.005 & 0.004 & 0.011 \\
\hline S I & 0.250 & 0.028 & 0.066 & 0.072 & 0.282 & 0.057 & 0.076 & 0.095 & 0.032 & 0.029 & 0.064 & 0.071 \\
\hline $\mathrm{K}_{\mathrm{I}}$ & -0.009 & 0.025 & 0.103 & 0.107 & -0.069 & 0.038 & 0.117 & 0.124 & -0.061 & 0.020 & 0.098 & 0.101 \\
\hline $\mathrm{Ca} \mathrm{I}$ & 0.020 & 0.012 & 0.025 & 0.029 & 0.054 & 0.010 & 0.030 & 0.033 & 0.034 & 0.009 & 0.025 & 0.028 \\
\hline Sc I & 0.076 & 0.031 & 0.056 & 0.065 & 0.072 & 0.089 & 0.062 & 0.109 & -0.004 & 0.058 & 0.051 & 0.078 \\
\hline Sc II & 0.079 & 0.012 & 0.030 & 0.034 & 0.062 & 0.010 & 0.033 & 0.036 & -0.018 & 0.013 & 0.028 & 0.032 \\
\hline $\mathrm{Ti} \mathrm{I}$ & 0.143 & 0.012 & 0.014 & 0.021 & 0.164 & 0.013 & 0.016 & 0.023 & 0.021 & 0.008 & 0.014 & 0.018 \\
\hline Ti II & 0.122 & 0.014 & 0.036 & 0.040 & 0.145 & 0.018 & 0.039 & 0.044 & 0.023 & 0.008 & 0.033 & 0.035 \\
\hline V I & 0.162 & 0.016 & 0.025 & 0.031 & 0.155 & 0.022 & 0.029 & 0.038 & -0.008 & 0.016 & 0.024 & 0.030 \\
\hline $\mathrm{Cr} \mathrm{I}$ & 0.026 & 0.013 & 0.015 & 0.021 & 0.030 & 0.015 & 0.017 & 0.025 & 0.004 & 0.008 & 0.014 & 0.019 \\
\hline Cr II & -0.018 & 0.031 & 0.075 & 0.082 & 0.004 & 0.105 & 0.084 & 0.134 & 0.021 & 0.074 & 0.071 & 0.103 \\
\hline Mn I & 0.115 & 0.025 & 0.062 & 0.067 & 0.176 & 0.038 & 0.071 & 0.081 & 0.061 & 0.020 & 0.059 & 0.063 \\
\hline Co I & 0.184 & 0.035 & 0.025 & 0.044 & 0.200 & 0.048 & 0.029 & 0.057 & 0.017 & 0.021 & 0.024 & 0.033 \\
\hline $\mathrm{Ni} I$ & 0.031 & 0.006 & 0.004 & 0.011 & 0.040 & 0.007 & 0.004 & 0.013 & 0.008 & 0.005 & 0.003 & 0.011 \\
\hline $\mathrm{Cu} \mathrm{I}$ & 0.235 & 0.025 & 0.062 & 0.067 & 0.226 & 0.038 & 0.071 & 0.081 & -0.009 & 0.020 & 0.059 & 0.063 \\
\hline $\mathrm{Zn} \mathrm{I}$ & 0.208 & 0.041 & 0.027 & 0.050 & 0.209 & 0.025 & 0.031 & 0.041 & 0.000 & 0.016 & 0.026 & 0.032 \\
\hline Sr I & 0.129 & 0.025 & 0.110 & 0.113 & 0.144 & 0.038 & 0.127 & 0.133 & 0.015 & 0.020 & 0.104 & 0.106 \\
\hline Y II & -0.004 & 0.048 & 0.039 & 0.063 & 0.083 & 0.089 & 0.045 & 0.100 & 0.086 & 0.043 & 0.037 & 0.057 \\
\hline $\mathrm{Ba} I \mathrm{I}$ & -0.105 & 0.088 & 0.044 & 0.099 & -0.031 & 0.115 & 0.050 & 0.126 & 0.074 & 0.029 & 0.042 & 0.051 \\
\hline La II & 0.095 & 0.025 & 0.078 & 0.083 & 0.086 & 0.038 & 0.086 & 0.094 & -0.009 & 0.020 & 0.073 & 0.076 \\
\hline Ce II & -0.130 & 0.025 & 0.077 & 0.081 & -0.217 & 0.038 & 0.084 & 0.092 & -0.088 & 0.020 & 0.073 & 0.076 \\
\hline
\end{tabular}

Notes. We also present the observational errors $\sigma_{\mathrm{obs}}$, errors due to stellar parameters $\sigma_{\mathrm{par}}$, as well as the total error $\sigma_{\mathrm{TOT}}$.

Table 2. Refined stellar and planetary parameters derived in this work.

\begin{tabular}{lcc}
\hline \hline Stellar parameters & Star A & Star B \\
\hline$T_{\text {eff }}(\mathrm{K})$ & $5364 \pm 57$ & $5190 \pm 58$ \\
$\log g(\mathrm{dex})$ & $4.39 \pm 0.18$ & $4.30 \pm 0.20$ \\
{$[\mathrm{Fe} / \mathrm{H}](\mathrm{dex})$} & $+0.016 \pm 0.009$ & $+0.022 \pm 0.010$ \\
$v_{\text {turb }}\left(\mathrm{km} \mathrm{s}^{-1}\right)$ & $0.79 \pm 0.12$ & $0.58 \pm 0.15$ \\
$M_{\star}\left(M_{\odot}\right)$ & $0.888 \pm 0.018$ & $0.861 \pm 0.015$ \\
$R_{\star}\left(R_{\odot}\right)$ & $0.910 \pm 0.009$ & $0.865 \pm 0.015$ \\
$\tau_{\star}(\mathrm{Gyr})$ & $9.233 \pm 2.133$ & $9.155 \pm 2.199$ \\
$\rho_{\star}\left(\mathrm{g} \mathrm{cm}{ }^{-3}\right)$ & $1.66 \pm 0.05$ & $1.88 \pm 0.06$ \\
\hline Planetary parameters & & \\
\hline$m_{\mathrm{p}} \sin i\left(M_{\text {Jup }}\right)$ & $9.08 \pm 0.20$ & \\
\hline
\end{tabular}

natal environments (see e.g. Tayouchi \& Chiba 2014; Mollá et al. 2015, and references therein). On the other hand, we discard GCE effects when mutually comparing stars A and B (owing to their common natal environment), this being an important advantage of the differential method. We corrected GCE effects for (A - Sun) and (B - Sun) by adopting the fitting trends of González Hernández et al. (2013), with a procedure similar to previous works (e.g. Liu et al. 2014; Saffe et al. 2015). Differential abundances are shown with filled points in Figs. 4 and 5, while the two dashed lines are weighted linear fits to

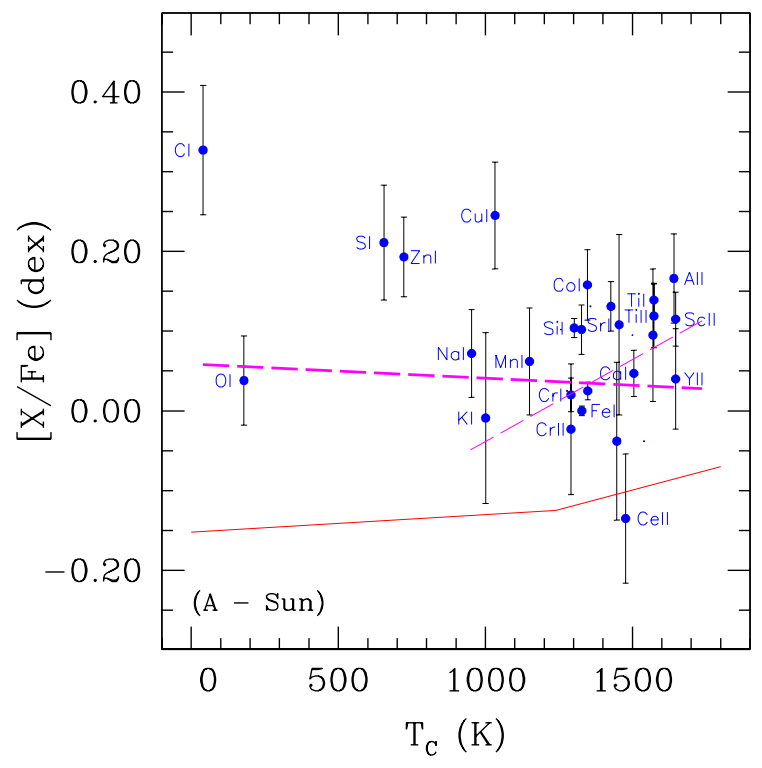

Fig. 4. Differential abundances (A - Sun) versus condensation temperature $T_{\mathrm{c}}$. Dashed lines are weighted linear fits to all and to refractory species, while continuous red lines show the solar-twins trend of M09 (vertically shifted for comparison).

all abundance values and only to the refractory species. We used as weight for each chemical element the inverse of the total abundance error $\sigma_{\mathrm{TOT}}$. We also included the solar-twins 


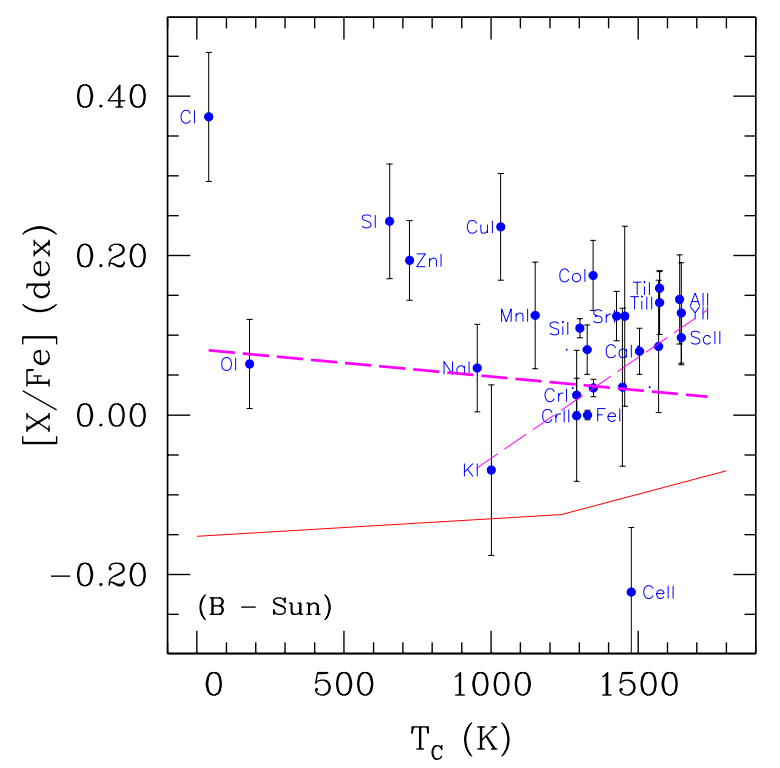

Fig. 5. Differential abundances (B - Sun) versus condensation temperature $T_{\mathrm{c}}$. Dashed lines are weighted linear fits to all and to refractory species, while continuous red lines show the solar-twins trend of M 09 (vertically shifted for comparison).

trend of M 09 using a continuous red line, vertically shifted for comparison.

In this work, we consider as a tentative trend those fits where the slope ranges between 2 and $3 \sigma_{\text {slope }}$, and a significant trend when the slope is greater than $3 \sigma_{\text {slope }}$. The general $T_{\mathrm{c}}$ fit to all species shown in Fig. 4 for the A star presents a slightly negative although non-significant slope $\left(-1.76 \pm 2.67 \times 10^{-5} \mathrm{dex} \mathrm{K}{ }^{-1}\right)$ when compared for example to the solar-twins trend of M 09 . The average abundance of the volatile species $\left(T_{\mathrm{c}}<900 \mathrm{~K}\right)$ is $\sim 0.22$ dex, while the average abundance of the refractory species $\left(T_{\mathrm{c}}>900 \mathrm{~K}\right)$ is $\sim 0.07 \mathrm{dex}$. On the other hand, the refractory species taken alone do show a clear positive trend (slope of $+20.6 \pm 4.60 \times 10^{-5} \mathrm{dex} \mathrm{K}^{-1}$ ). This corresponds to an excess in refractories when compared to the Sun (which would display a horizontal tendency, not shown) and also when compared to the solar-twins trend of M09 (red continuous line). Star B shows in Fig. 5 similar behaviour to that shown by the A star in the Fig. 4: a slightly negative although non-significant general slope $\left(-3.46 \pm 3.06 \times 10^{-5}\right.$ dex $\left.\mathrm{K}^{-1}\right)$ together with a positive trend for refractories (slope of $+25.3 \pm 5.29 \times 10^{-5} \mathrm{dex} \mathrm{K}^{-1}$ ). Therefore, following a reasoning similar to M 09, stars A and B do not seem to be depleted in refractory elements when compared to the solar twins, which is different for the case of the Sun. In other words, terrestrial planet formation would have been less efficient in the stars of this binary system than in the Sun.

The differential abundances of star B using A as reference (B - A) are presented in Fig. 6. This plot corresponds to the abundance values derived with the highest possible precision, diminishing errors in the calculation of stellar parameters and GCE effects (e.g. Saffe et al. 2015).

The average differential abundance of the volatile species is $\sim 0.026$ dex, while the average of the refractories amounts to $\sim 0.005$ dex, showing no clear general trend within the errors (slope of $+0.47 \pm 2.35 \times 10^{-5} \mathrm{dex} \mathrm{K}^{-1}$ ). The refractory elements

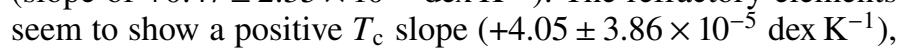
however there is no significant trend due to the relatively large dispersion of the slope. We consider that data with higher quality

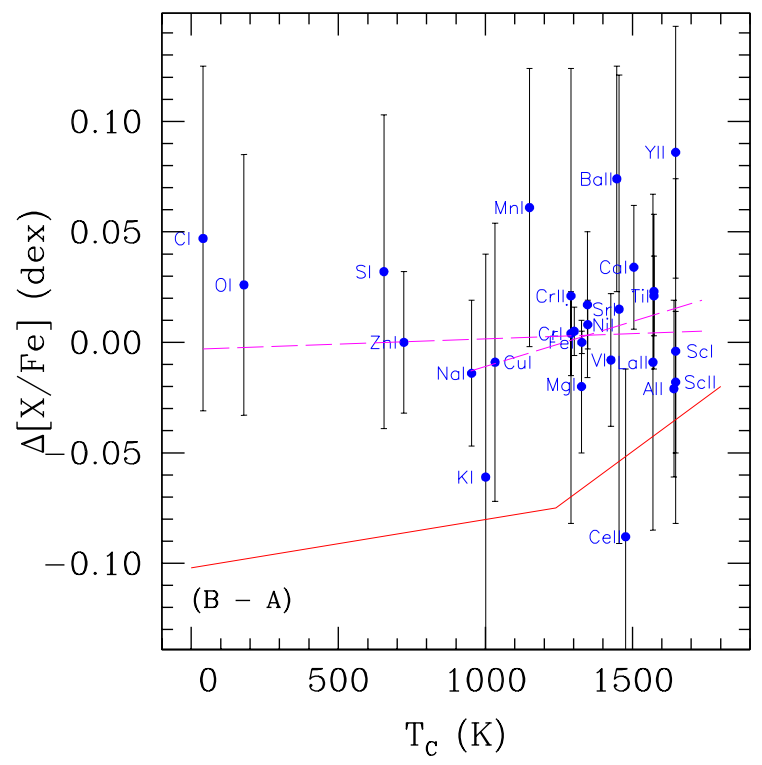

Fig. 6. Differential abundances $(B-A)$ versus condensation temperature $T_{\mathrm{c}}$. Long-dashed lines are a weighted linear fit to all and to the refractory species. The solar-twins trend of M09 is shown with a continuous red line (vertically shifted for comparison).

(perhaps higher $\mathrm{S} / \mathrm{N}$ ) is desirable, because there may be a hidden trend with $T_{\mathrm{c}}$ that the current data cannot discern (average error bars of $\sim 0.05 \mathrm{dex})$. Following our results, the difference in metallicity for $(\mathrm{B}-\mathrm{A})$ is only $+0.006 \pm 0.009 \mathrm{dex}$, that is, both stars present almost the same metallicity within our errors. Therefore, both stars present very similar metallicity, and there is no clear difference in the relative content of refractory and volatile elements within our errors. In other words, there is no clear $T_{\mathrm{c}}$ trend between stars $\mathrm{A}$ and $\mathrm{B}$, and therefore no clear evidence of terrestrial planet formation in this binary system. Similarly, Liu et al. (2014) concluded that the presence of a giant planet does not necessarily introduce a terrestrial (or rocky) chemical signature into their host stars, by studying the HAT-P-1 binary system. In our case, the massive planet orbiting the A star of the HD 106515 binary system presents a relatively high eccentricity (0.57, Desidera et al. 2012; Marmier et al. 2013). The presence of long-period planets with eccentric orbits was noted by Marmier et al. (2013), who included HD 106515 in their analysis and attributed the origin of the eccentricity to a possible Kozai mechanism. For the case of eccentric giant planets, numerical simulations also found that the early dynamical evolution of giant planets clears out most of the possible terrestrial planets in the inner zone (Veras \& Armitage 2005, 2006; Raymond et al. 2011).

We derived stellar parameters for stars A and B by applying both the classical solar-scaled method and the new non-solarscaled procedure (Saffe et al. 2018). The $\Delta$ differences in the parameters taken as (new method - classical method) amount to $\Delta T_{\text {eff }}+27 \mathrm{~K}, \Delta \log g+0.02 \mathrm{dex}, \Delta[\mathrm{Fe} / \mathrm{H}]+0.018 \mathrm{dex}$, and $\Delta v_{\text {turb }}+0.07 \mathrm{~km} \mathrm{~s}^{-1}$ for the A star, while for the B star they amount to $\Delta T_{\text {eff }}+23 \mathrm{~K}, \Delta \log g+0.02 \mathrm{dex}, \Delta[\mathrm{Fe} / \mathrm{H}]+0.012 \mathrm{dex}$, and $\Delta v_{\text {turb }}-0.34 \mathrm{~km} \mathrm{~s}^{-1}$. Therefore, for the case of the classical solar-scaled method, we should include in the total error estimation of these parameters a quantity similar to $\Delta$. In this way, considering for example the $T_{\text {eff }}$ of stars $\mathrm{A}$ and $\mathrm{B}$ with classical errors of 57 and $58 \mathrm{~K}$, and adding quadratically the $\Delta$ differences of 27 and $23 \mathrm{~K}$, would result in a final total error of 63 and $62 \mathrm{~K}$ for stars A and B. In other words, for the stars of this binary 


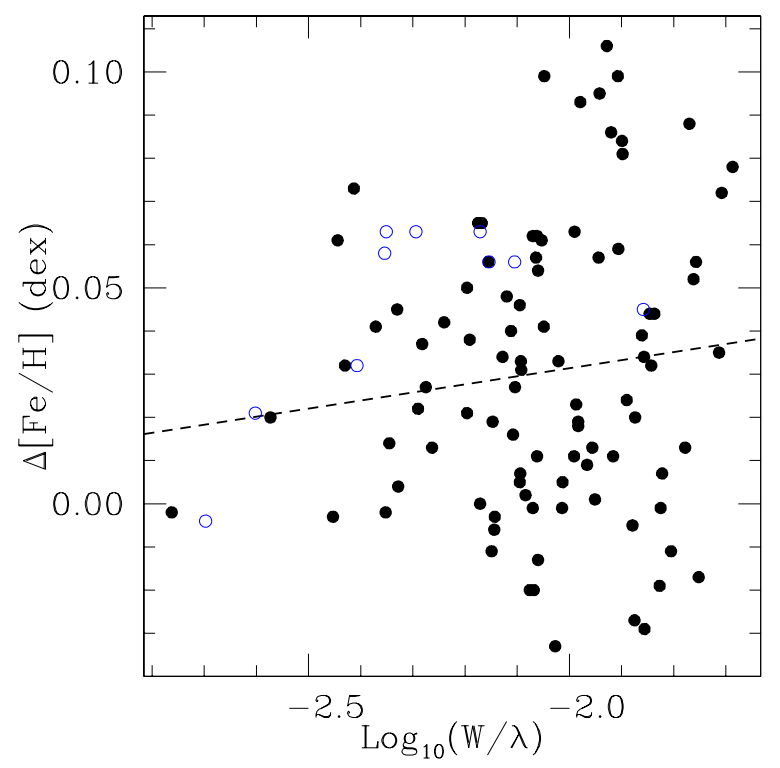

Fig. 7. Differential abundances (A - Sun) versus excitation potential using non-solar-scaled opacities, but forced to the solar-scaled solution. Filled and empty points correspond to Fe I and Fe II, respectively. The dashed line is a linear fit to the abundance values.

system, the use of the new method allows a reduction of $\sim 10 \%$ of the total error in $T_{\text {eff }}$.

We note that the new method allows an improvement in the calculation of stellar parameters, while NLTE or GCE corrections only affect the chemical abundances. We present in the Fig. 7 iron abundances versus $\mathrm{EW}_{\mathrm{r}}$, using non-solar-scaled opacities but using the solution found in the solar-scaled method. Filled and empty points correspond to Fe I and Fe II, respectively, while the dashed line is a linear fit to the abundance values. Both the presence of an unbalance in this plot, and the fact that $\mathrm{Fe}$ II values are greater than Fe I values, shows that the solar-scaled solution (requiring e.g. the excitation and ionization balance of iron lines) is not compatible with the new method. This shows that we can indeed derive a refined solution in stellar parameters when using non-solar-scaled opacities.

We present in Fig. 8 differential abundances versus atomic number using the solar-scaled method (blue circles and lines) and using the new method (red circles and lines) for star B. The same plot for the A star shows a similar behaviour. In the lower panel we present using bars the difference between both procedures (as new method - solar-scaled method), for each chemical species. Some chemical elements are labelled in the plot to facilitate their identification. The lower panel can be considered as an abundance pattern difference obtained when using one method or another. Similar to Saffe et al. (2018), we note that C, O, and $\mathrm{S}$ present lower values when using the new method, while the rest of the species mostly present similar or greater abundance values, by an average of $\sim 0.015 \mathrm{dex}$. The greater difference for star B corresponds to La, with a difference near $\sim 0.04$ dex. We also present in Fig. 9 a plot similar to Fig. 8, however, in this case for $(\mathrm{B}-\mathrm{A})$. Due to the physical similarity between stars $\mathrm{A}$ and $\mathrm{B}$, the differences in the individual abundances are usually lower than those of Fig. 8. Most chemical species show almost negligible differences (see lower panel), while some elements show differences of up to $\sim 0.010 \operatorname{dex}$ (for $\mathrm{O}, \mathrm{K}, \mathrm{V}, \mathrm{Sr}$, and $\mathrm{Y}$ ). Therefore, the differences between both methods are comparable to NLTE corrections or GCE effects and cannot be easily ignored, especially when comparing the stars using

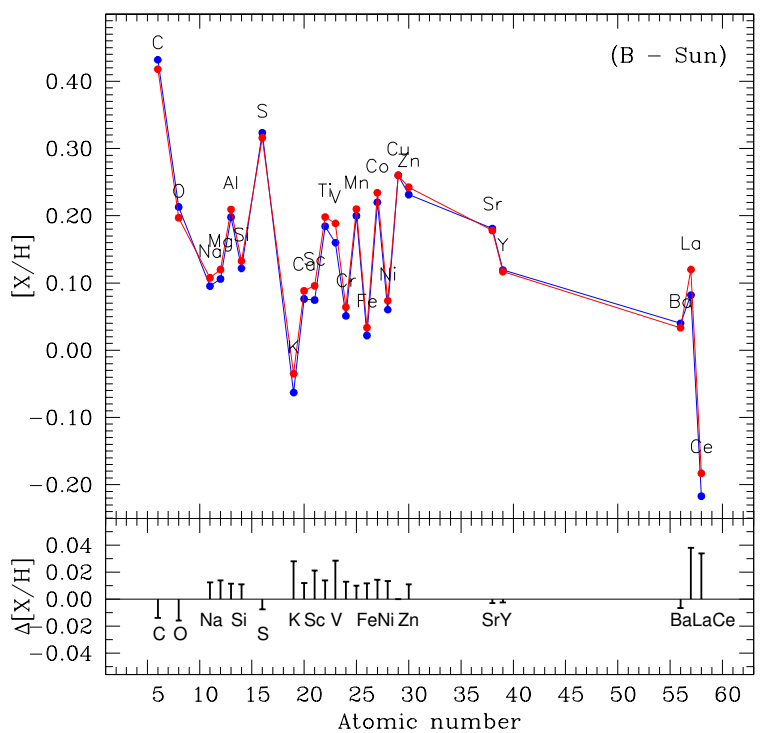

Fig. 8. Differential abundances (B - Sun) versus atomic number. Red circles and lines correspond to the solar-scaled method, while blue circles and lines corresponds to the new method. The lower panel presents with bars the difference between both procedures (as new method solar-scaled method).

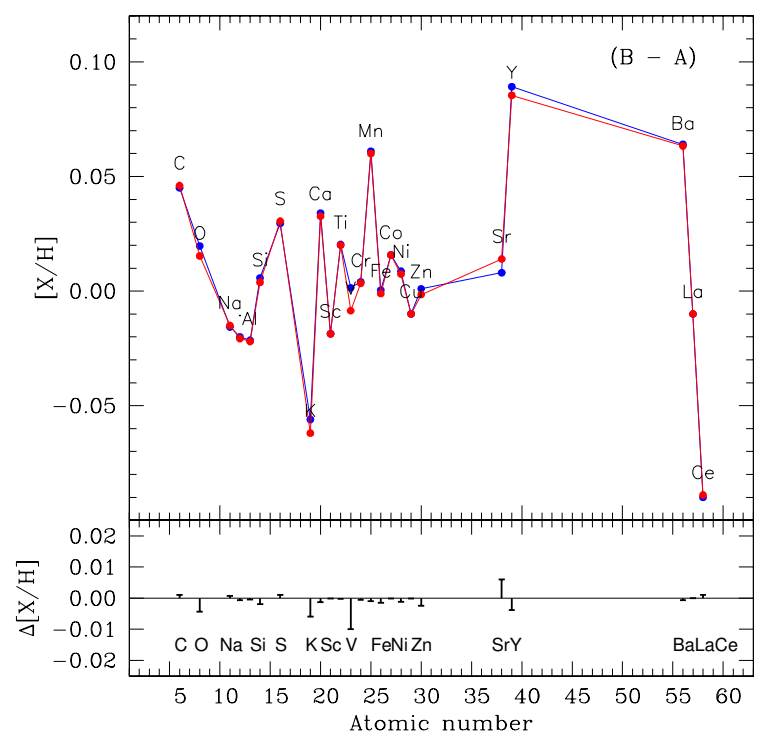

Fig. 9. Differential abundances (B - A) vs. atomic number. Red circles and lines correspond to the solar-scaled method, while blue circles and lines corresponds to the new method. The lower panel presents with bars the difference between both procedures (as new method - solarscaled method).

the Sun as reference rather than comparing between the stars themselves.

We explored the possibility to use "corrected" solar-scaled models rather than the full non-solar-scaled approach for star B by recomputing solar-scaled models but using a modified metallicity. We used a corrected metallicity similar to Eq. (3) of Salaris et al. (1993): $\delta[\mathrm{Fe} / \mathrm{H}]=\log _{10}\left(0.638 \times 10^{[\alpha / \mathrm{Fe}]}+0.362\right)$, where $\delta[\mathrm{Fe} / \mathrm{H}]$ is the amount of the correction and $[\alpha / \mathrm{Fe}]$ is the average of the abundances of the alpha elements. For star B we estimated $[\alpha / \mathrm{Fe}] \sim 0.155$ dex and $\delta[\mathrm{Fe} / \mathrm{H}] \sim 0.104$ dex. The resulting abundances for this correction are presented in Fig. 10. We note in this plot (see e.g. the lower panel) that there is not a perfect 


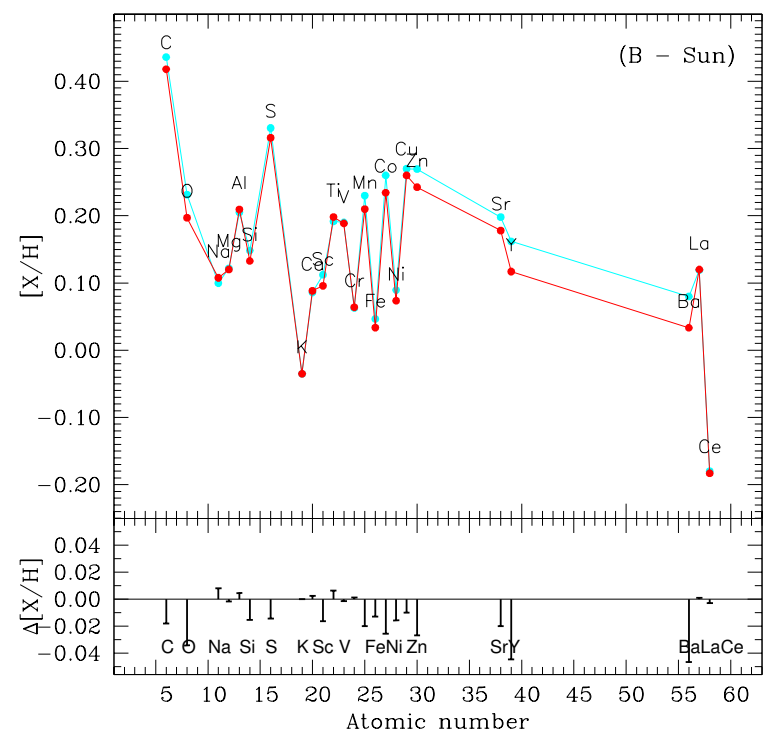

Fig. 10. Differential abundances $(B$ - Sun) versus atomic number. Red circles and lines correspond to the non-solar-scaled method, while cyan circles and lines correspond to the other "corrected" solar-scaled method. The lower panel presents with bars the difference between both procedures (as non-solar-scaled - corrected solar-scaled method).

match between the abundance values derived with the non-solarscaled and corrected solar-scaled methods. We suspect that this is due, at least in part, to the fact that solar-scaled models even with corrected $[\mathrm{Fe} / \mathrm{H}]$ values still made use of solar-scaled opacities when deriving abundances of chemical species other than $\mathrm{Fe}$, giving rise possibly to the small differences observed in Fig. 10 .

\section{Conclusions}

Aiming to detect a possible chemical signature of planet formation, we determined the stellar parameters and chemical pattern in both components of the notable binary system HD 106515 , with the highest possible precision. Star A hosts a massive longperiod planet while there is no planet detected around star B. The strong similarity between both stars greatly diminishes errors in the abundance determination, GCE, or evolutionary effects. We started by deriving the parameters for stars A and B using the Sun as reference, $(\mathrm{A}-\mathrm{Sun})$ and $(\mathrm{B}-\mathrm{Sun})$, and then we recomputed the parameters for the $\mathrm{B}$ star using $\mathrm{A}$ as reference, (B - A), obtaining the same results. We derived very similar chemical patterns for stars A and B. By studying the possible temperature condensation $T_{\mathrm{c}}$ trends, we concluded that the stars do not seem to be depleted in refractory elements, which differs from the case of the Sun (Meléndez et al. 2009). However, we suggest that data with higher quality (perhaps higher $\mathrm{S} / \mathrm{N}$ ) are desirable, because there may be a hidden trend with $T_{\mathrm{c}}$ that the current data cannot discern (average error bars of $\sim 0.05 \mathrm{dex}$ ). Then, following the reasoning of Meléndez et al. (2009), the terrestrial planet formation would have been less efficient in the stars of this binary system than in the Sun. In comparing the stars to each other, the lack of clear $T_{\mathrm{c}}$ trend implies that the presence of a giant planet does not necessarily imprint a (terrestrial) chemical signature on its host star, similar to previous results (Liu et al. 2014; Saffe et al. 2015). We note, however, that the A star is orbited by a massive eccentric planet, where numerical simulations found that the early dynamical evolution of giant planets clears out most of the possible terrestrial planets in the inner zone (Veras \& Armitage 2005, 2006; Raymond et al. 2011). In this way, both binary systems HD 80606 and HD 106515 do not seem to present a (terrestrial) signature of planet formation (Saffe et al. 2015), with both systems hosting an eccentric giant planet.

For both stars in the binary system, we refined the stellar mass, radius, and age. In particular, we found a notable difference of $\sim 78 \%$ in the stellar radius of HD 106515 A compared to the value of Marmier et al. (2013). This difference would seriously affect the derived planetary properties (radius, density) of a potential transiting planet that could be detected by the Transiting Exoplanet Survey Satellite (TESS) mission in the next months. In addition, we refined the minimum planetary mass to $m_{\mathrm{p}} \sin i=9.08 \pm 0.20 M_{\mathrm{Jup}}$, which differs by $\sim 6 \%$ when compared with the value obtained by Marmier et al. (2013).

We also take the opportunity to compare the parameters derived with non-solar-scaled and classical solar-scaled methods. We obtained a small but noticeable difference in stellar parameters and individual chemical patterns. We showed that using non-solar-scaled opacities, the classical solution cannot verify the standard excitation and ionization balance of iron, similar to Saffe et al. (2018). Also, the difference in abundances between both procedures is comparable to NLTE or GCE effects, especially when using the Sun as reference, and cannot be easily avoided in high-precision studies. Therefore, we encourage the use of non-solar-scaled opacities in studies that require the highest possible precision, such as the detection of a possible chemical signature of planet formation in a binary system.

Acknowledgements. We thank the anonymous referee for constructive comments that improved the paper. The authors wish to recognize and acknowledge the very significant cultural role and reverence that the summit of Mauna Kea has always had within the indigenous Hawaiian community. We are most fortunate to have the opportunity to conduct observations from this mountain. M.F. and F.M.L. acknowledge the financial support from CONICET in the form of Post-Doctoral Fellowships. The authors also thank Drs. R. Kurucz, C. Sneden, and L. Girardi for making their codes available to us.

\section{References}

Adibekyan, V., González Hernández, J., Delgado-Mena, E., et al. 2014, A\&A, 564, A 15

Adibekyan, V., Delgado-Mena, E., Figueira, P., et al. 2016, A\&A, 592, A87

Bedell, M., Meléndez, J., Bean, J. L., et al. 2014, ApJ, 795, 23

Bensby, T., Feltzing, S., \& Lundström, I. 2004, A\&A, 415, 155

Bressan, A., Marigo, P., Girardi, L., et al. 2012, MNRAS, 427, 127

Cumming, A., Marcy, G. W., Butler, R. P. 1999, ApJ, 526, 890

da Silva, L., Girardi, L., Pasquini, L., et al. 2006, A\&A, 458, 609

Desidera, S., Gratton, R. G., Scuderi, S., et al. 2004, A\&A, 420, 683

Desidera, S., Gratton, R. G., Lucatello, S., \& Claudi, R. U. 2006, A\&A, 454 581

Desidera, S., Gratton, R., Carolo, E., et al. 2012, A\&A, 546, A118

ESA 1997, VizieR Online Data Catalog: I/239

Gaia Collaboration (Brown, A. G. A., et al.) 2018, A\&A, 616, A1

Girardi, L., Bressan, A., Bertelli, G., \& Chiosi, C. 2000, VizieR Online Data Catalog: J/A+AS/141/371

González Hernández, J., Delgado Mena, E., Sousa, S. G., et al. 2013, A\&A, 552, A6

Høg, E., Fabricius, C., Makarov, V. V., et al. 2000, A\&A, 355, L27

Johansson, S., Litzén, U., Lundberg, H., \& Zhang, Z. 2016, ApJ, 584, L107

Korotin, S. A., Andrievsky, S., Hansen, C., et al. 2015, A\&A, 581, A70

Kurucz, R. L. 1993, ATLAS9 Stellar Atmosphere Programs and $2 \mathrm{~km} \mathrm{~s}^{-1}$ grid, Kurucz CD-ROM No. 13 (Cambridge, MA: Smithsonian Astrophysical Observatory)

Kurucz, R., \& Bell, B. 1995, Atomic Line Data, Kurucz CD-ROM No. 23, (Cambridge, MA: Smithsonian Astrophysical Observatory)

Lambert, D. L. 1978, MNRAS, 182, 249

Laws, C., \& Gonzalez, G. 2016 ApJ, 553, 405L

Liu, F., Asplund, M., Ramírez, I., Yong, D., \& Meléndez, J. 2014, MNRAS, 442, L51 
C. Saffe et al.: High-precision analysis of binary systems

Lodders, K. 2003, AJ, 591, 1220

Mack, C., Schuler, S., Stassun, K., et al. 2014, ApJ, 787, 98

Marmier, M., Segransan, D., Udry, S., et al. 2013, A\&A, 551, A90

Mayor, M., Marmier, M., Lovis, C., et al. 2011, ArXiv e-prints [arXiv:1109.2497]

Meléndez, J., Asplund, M., Gustafsson, B., \& Yong, D. 2009, AJ, 704, L66

Meléndez, J., Ramírez, I., Karakas, A., et al. 2014, AJ, 791, 14

Mollá, M., Cavichia, O., \& Gibson, B. 2015, MNRAS, 451, 3693

Nissen, P. E., Silva Aguirre, V., Christensen-Dalsgaard, J., et al. 2017, A\&A, 608, A112

Ramírez, I., Allende Prieto, C., \& Lambert, D. 2007, A\&A, 465, 271

Ramírez, I., Meléndez, J., \& Asplund, M. 2009, A\&A, 508, L17

Ramírez, I., Asplund, M., Baumann, P., Meléndez, J., \& Bensby, T. 2010, A\&A, 521, A33

Ramírez, I., Meléndez, J., Cornejo, D., Roederer, I., \& Fish, J. 2011, AJ, 740, 76

Raymond, S., Armitage, P., Moro-Martín, A., et al. 2011, A\&A, 530, A62

Saffe, C. 2011, Rev. Mex. Astron. Astrofis., 47, 3
Saffe, C., Flores, M., \& Buccino, A. 2015, A\&A, 582, A17

Saffe, C., Flores, M., Jaque Arancibia, M., Buccino, A., \& Jofré, E. 2016, A\&A, 588, A81

Saffe, C., Jofré, E., Martioli, E., et al. 2017, A\&A, 604, L4

Saffe, C., Flores, M., Miquelarena, P., et al. 2018, A\&A, 620, A54

Salaris, M., Chieffi, A., \& Straniero, O. 1993, ApJ, 414, 580

Schuler, S., Cunha, K., Smith, V., et al. 2011, ApJ, 737, L32

Shi, J. R., Gehren, T., \& Zhao, G. 2004, A\&A, 423, 683

Sneden, C. 2016, ApJ, 184, 839

Takeda, Y. 2005, PASJ, 57, 83

Tayouchi, D., \& Chiba, M. 2014, AJ, 788, 89

Teske, J., Schectman, S., Vogt, S., et al. 2016a, AJ, 152, 167

Teske, J., Khanal, S., \& Ramírez, I. 2016b, ApJ, 819, 19

Tucci Maia, M., Meléndez, J., \& Ramírez, I. 2014, ApJ, 790, L25

Veras, D., \& Armitage, P. 2005, ApJ, 620, L111

Veras, D., \& Armitage, P. 2006, ApJ, 645, 1509

Vogt, S. S., Allen, S. L., Bigelow, B. C., et al. 1994, SPIE, 2198, 362 